\title{
Effectiveness of Duloxetine for Postsurgical Chronic Neuropathic Disorders after Spine and Spinal Cord Surgery
}

\author{
Osahiio Tsuji ${ }^{1}$, Shizuko Kosugi ${ }^{2}$, Satoshi Suzuki ${ }^{1}$, Satoshi Nori ${ }^{1}$, Narihito Nagoshi ${ }^{1}$, Eijiro Okada ${ }^{1}$,
} Nobuyuki Fujita ${ }^{3}$, Mitsuru Yagi ${ }^{1}$, Masaya Nakamura ${ }^{1}$, Morio Matsumoto ${ }^{1}$, Kota Watanabe ${ }^{1}$

${ }^{I}$ Department of Orthopaedic Surgery, Keio University School of Medicine, Tokyo, Japan

${ }^{2}$ Department of Anesthesiology, Keio University School of Medicine, Tokyo, Japan

${ }^{3}$ Department of Orthopaedic Surgery, Fujita Health University, Aichi, Japan

Study Design: This is a retrospective observational study with an outpatient setting.

Purpose: This study aimed to describe the effects of duloxetine (DLX) administration for postsurgical chronic neuropathic disorders (both pain and numbness) following spinal surgery in patients without depression.

Overview of Literature: Although several reports indicated the potential of DLX to effectively treat postoperative symptoms as a perioperative intervention, there have been no reports of its positive effect on postsurgical chronic neuropathic disorders.

Methods: A total of 24 patients with postsurgical chronic pain and/or numbness Numeric Rating Scale (NRS) scores of $\geq 4$ were enrolled. All patients underwent spine or spinal cord surgery at Keio University Hospital and received daily administration of DLX for more than 3 months. The mean postoperative period before the first administration of DLX was $35.5 \pm 57.0$ months. DLX was administered for more than 3 months at a dose of 20, 40, or $60 \mathrm{mg} /$ day, and the degree of pain and numbness was evaluated using the NRS before administration and 3 months after administration. Effectiveness was defined as more than a 2-point decrease in the NRS score following administration.

Results: In terms of the type of symptoms, 15 patients experienced only numbness, eight experienced both pain and numbness, and one experienced only pain. Of the 24 patients, 19 achieved effective relief with DLX. DLX was effective for all patients with postsurgical chronic pain $(n=9)$, and it reduced postsurgical chronic numbness in 18 of 23 patients. No significant difference was observed in background spinal disorders. DLX was not effective for five patients who complained only of postsurgical chronic numbness.

Conclusions: This study reports the effectiveness of DLX for postsurgical chronic neuropathic disorders. Although DLX reduced postsurgical chronic pain (efficacy rate $=100 \%$ ) and numbness $(78.3 \%$ ) in certain patients, further investigation is needed to determine its optimal use.

Keywords: Duloxetine hydrochloride; Chronic pain; Numbness; Postsurgical neuropathic disorders

Received Apr 24, 2020; Revised May 25, 2020; Accepted Jun 20, 2020

Corresponding author: Kota Watanabe

Department of Orthopaedic Surgery, Keio University School of Medicine, 35 Shinanomachi, Shinjuku-ku, Tokyo, 160-8582, Japan

Tel: +81-3-5363-3812, Fax: +81-3-3353-6597, E-mail: kw197251@keio.jp 


\section{Introduction}

In recent years, the development of minimally invasive surgical techniques for spine and spinal cord disorders has improved surgical outcomes, especially in terms of perioperative and postoperative pain. However, neuropathic pain following spine surgery, which is sometimes referred to as failed back surgery syndrome, remains a major problem, and residual numbness after surgery for lumbar spinal stenosis (LSS) is especially scarcely settled. Chronic postoperative residual pain and numbness (postsurgical chronic pain and numbness) are a nuisance for spine surgeons, and it is generally agreed that preoperative numbness at rest is particularly refractory [1]. Postsurgical chronic pain following spine surgery is heavily influenced by neuropathic pain, which is characterized not only by spontaneous and provoked pain but also by paresthesia and dysesthesias reflecting neural damage [2]. Only gabapentinoids have been reported to effectively treat neuropathic pain following spine surgery [3], and a multidisciplinary treatment approach is needed [4].

Although the precise mechanisms of central pain inhibition to facilitate the descending pain inhibitory pathway remain uncertain, duloxetine (DLX), a serotonin and norepinephrine reuptake inhibitor (SNRI) that mediates pain inhibition in the descending pathways, has attracted much attention for evidence as an analgesic agent for diabetic peripheral neuropathic pain [5], fibromyalgia [6], chronic low back pain [7], and pain associated with knee osteoarthritis [8] and for the treatment of psychiatric diseases, such as depression and anxiety disorders. Recently, several previous reports have indicated that DLX could reduce morphine requirements following knee replacement surgery [9] and opioid consumption following spine surgery [10]. Furthermore, DLX could improve the outcomes of spinal cord stimulation for chronic pain [11], and perioperative administration of DLX combined with etoricoxib, a cyclooxygenase- 2 inhibitor and nonsteroidal anti-inflammatory drug, could improve analgesia and reduce opioid consumption after lumbar laminectomy [12]. Collectively, the clinical utility of DLX for spine surgery is beyond dispute. Abovementioned reports have addressed the effectiveness of DLX prescribed within 48 hours after surgery on postoperative pain. Based on the analogy of this previously reported effectiveness of DLX on postoperative pain, DLX prescription was supposed to produce sustainable effects on persistent chronic pain after surgery.
This study aimed to retrospectively examine the effects of DLX on persistent chronic postsurgical symptoms, including both pain and numbness, after spine and spinal cord surgery in patients without depression or other mental disorders.

\section{Materials and Methods}

\section{Study design and setting}

This was a retrospective observational study evaluating the efficacy of DLX on persistent chronic postsurgical neuropathic disorders (i.e., pain and numbness) in an outpatient setting. After obtaining Institutional Review Board approval, we retrospectively reviewed the medical records of patients who underwent spinal surgery at Keio University Hospital and who received DLX after December 2016 for the treatment of chronic low back pain or knee pain associated with osteoarthritis in an outpatient clinic. Dopamine D2 receptor antagonist (e.g., domperidone, sulpiride, and itopride) or selective serotonin $5-\mathrm{HT}_{4}$ receptor agonist (mosapride) was administered, depending on each individual's degree of side effect, to mitigate the side effects of DLX, such as nausea and vomiting.

\section{Participants}

A total of 24 patients (15 males and nine females) who underwent spine or spinal cord surgery at our institution between 2002 and 2016 and who presented with residual symptoms (i.e., pain and/or numbness) at and/or below the lesion site for more than 3 months were enrolled in this study. The residual neuropathic disorders (pain and/ or numbness) were definitely diagnosed according to the grading system for the diagnosis of neuropathic pain, which was advocated by the Special Interest Group on Neuropathic Pain of the International Association for the Study of Pain [13,14]. Then, a Numeric Rating Scale (NRS) was used to asses residual neuropathic pain and numbness [15]; an NRS score of 0 was defined as no pain or numbness, and an NRS score of 10 was defined as the worst pain and/or numbness ever experienced. All patients had postsurgical chronic neuropathic disorders with an NRS score of $\geq 4$.

All patients had a history of pregabalin prescription regardless of its continuation. DLX was prescribed in all cases, which was initiated at $20 \mathrm{mg} /$ day and gradually 
increased to a maximum of $60 \mathrm{mg} /$ day, according to each patient's degree of adverse effects, such as nausea and dizziness. The dosage that provided adequate symptom relief was then maintained for a period of more than 3 months. Patients were allowed to continue analgesics or other previously administered concomitant medications during treatment with DLX. All patients presented to our institution's outpatient clinic for follow-up, which usually occurred every 3-5 weeks after the first visit. Patients were asked to rate each residual symptom using the NRS 3 months after the DLX treatment initiation with adequate symptom relief.

To compare the NRS results of postsurgical chronic pain and numbness before and 3 months after the DLX treatment initiation, patients with more than a 2-point decrease in the NRS score after administration ( $\triangle$ NRS score $\geq 2$ ) were classified as the effective group (E group) and the others as the not effective group ( $\mathrm{N}$ group), according to previous reports [15]. An NRS score of $\geq 7$ was defined as severe; 4-6, moderate; $1-3$, mild; and 0, none [16]. Furthermore, the degree of symptom (i.e., pain and numbness) relief was defined as follows: $a \geq 2$-point decrease in the pain or numbness intensity assessed using the NRS, which signified a clinically meaningful reduction, was defined as much improved, and a $>50 \%$ decrease in the NRS score than baseline or an NRS score of $\leq 2$ after 3 months of treatment was considered substantial symptom (pain and/or numbness) relief and was defined as very much improved [17]. A $\leq 1$-point decrease in the NRS score was defined as no change.

\section{Data collection}

The type of neuropathic disorder (i.e., pain only, numbness only, or both) was assessed in the outpatient clinic before the DLX administration. First, the efficacy rate of DLX for each neuropathic disorder (i.e., pain and numbness) was investigated 3 months after DLX was prescribed. Then, data for possible related factors for the effect of DLX on postoperative neuropathic disorders were collected, such as patient characteristics (i.e., age, gender, and body mass index $[\mathrm{BMI}])$, primary spinal disease that led to surgery, postoperative duration before DLX administration, maintenance dosage of DLX, concomitant tramadol and/or pregabalin use, and past medical history strongly related to neuropathy, such as type 2 diabetes mellitus.

\section{Statistical analysis}

Data are expressed as means \pm standard deviations. Pearson's chi-square test was used for categorical variables to assess the differences between the $\mathrm{E}$ and $\mathrm{N}$ groups. The Wilcoxon signed-rank test was used to conduct paired analyses, and the Mann-Whitney $U$-test was used to conduct unpaired analyses for continuous variables. Statistical analysis was performed using the IBM SPSS ver. 25.0 (IBM Corp., Armonk, NY, USA). A $p<0.05$ was considered statistically significant.

\section{Ethics approval and informed consent}

The study protocol was conducted in accordance with the Declaration of Helsinki and in compliance with ethical guidelines for medical and health research involving human subjects. Moreover, this study protocol was approved by the ethics committee of Keio University School of Medicine (approval no., 20110222). Informed consent was obtained in the form of opt-out on the website. Those who rejected were excluded.

\section{Results}

A total of 24 patients (15 males and nine females) were included in this study with a mean age of $67.3 \pm 11.1$ years (median, 73 years; range, 48-85 years) (Table 1). No significant difference was observed in the geographic background data (i.e., age, gender, and BMI) between the $\mathrm{E}$ and $\mathrm{N}$ groups. With regard to the primary disease that led

Table 1. General information on all participants

\begin{tabular}{|c|c|}
\hline Characteristic & Total value \\
\hline \multicolumn{2}{|l|}{ Gender } \\
\hline Male & 15 \\
\hline Female & 9 \\
\hline Mean age (yr) & $67.3 \pm 11.1$ \\
\hline Median age (yr) & $73(48-85)$ \\
\hline \multicolumn{2}{|l|}{ Primary disease } \\
\hline Degenerative & 14 \\
\hline Spinal cord & 10 \\
\hline Duration after surgery (mo) & $35.5 \pm 57.0(3-180)$ \\
\hline
\end{tabular}


to spinal surgery, there were 14 patients with degenerative spinal disease (10 with LSS and four with cervical spondylosis) and 10 patients with spinal cord disorders (Table 2). Of these 10 patients, three had cervical extramedullary schwannoma; two, cervical intramedullary tumors; two, lumbar extramedullary schwannoma; and three, other thoracic lesions (i.e., extramedullary schwannoma, spinal cord herniation, and dural arteriovenous fistula). The mean duration of the postoperative period was $35.5 \pm 57.0$ months (range, 3-180 months). There were 15 patients with only numbness, eight with both pain and numbness, and one with only pain.

A total of 19 patients (E group) had a change greater than 2 points in the NRS score after DLX initiation (efficacy rate $=79.2 \%$ ). DLX was effective in all patients with postsurgical chronic pain $(n=9$, efficacy rate $=100 \%)$ according to our definition ( $\triangle \mathrm{NRS} \geq 2$ ), whereas DLX reduced postsurgical chronic numbness in 18 of $23 \mathrm{pa}$ tients (efficacy rate $=78.3 \%$ ). All five of these patients were in the $\mathrm{N}$ group and exhibited only postsurgical chronic numbness, and the efficacy rates according to the disease

Table 2. Geographic data of participants in the study

\begin{tabular}{|c|c|c|c|}
\hline Characteristic & E-group $(n=19)$ & $N$-group $(n=5)$ & $p$-value \\
\hline Gender & & & NS \\
\hline Male & 12 & 3 & \\
\hline Female & 7 & 2 & \\
\hline Age (yr) & $66.9 \pm 11.2$ & $68.4 \pm 11.5$ & NS \\
\hline Background spinal disorder & & & NS \\
\hline Degenerative & 10 & 4 & \\
\hline Spinal cord & 9 & 1 & \\
\hline Height (cm) & $160.7 \pm 10.1$ & $159.8 \pm 8.0$ & NS \\
\hline Body weight $(\mathrm{kg})$ & $63.8 \pm 16.0$ & $58.0 \pm 10.3$ & NS \\
\hline Body mass index $\left(\mathrm{kg} / \mathrm{m}^{2}\right)$ & $24.4 \pm 4.0$ & $22.6 \pm 2.6$ & NS \\
\hline $\begin{array}{l}\text { Duration before duloxetine } \\
\text { administration (mo) }\end{array}$ & $50.5 \pm 60.8(3-180)$ & $45.8 \pm 44.6(10-120)$ & NS \\
\hline $\begin{array}{l}\text { Ongoing concomitant } \\
\text { pregabalin usage }\end{array}$ & 10 & 4 & NS \\
\hline $\begin{array}{l}\text { Ongoing concomitant } \\
\text { tramadol usage }\end{array}$ & 4 & 1 & NS \\
\hline Diabetes & 4 & 0 & 0.012 \\
\hline
\end{tabular}

Values are presented as number, mean $\pm S D$, or mean $\pm S D$ (range). Geographic data of patients in the E group ( $n=19)$, which were defined as those with more than a 2-point decrease in their NRS scores (each for pain and numbness) after duloxetine administration, and the $\mathrm{N}$ group $(n=5)$, which were defined as those with less than a 1-point decrease in the NRS score for pain or numbness. SD, standard deviation; NS, no significant difference; NSR, Numeric Rating Scale. category were $71.4 \%$ (10 of 14 ) for degenerative spinal diseases and 90\% (nine of 10) for spinal cord disorders. No significant difference was observed in the DLX efficacy rate of patients with degenerative spinal disease and those with spinal disorders (Table 2). The mean NRS pain scores ( $n=9$, all cases with postsurgical chronic pain were in the E group) significantly decreased after 3 months of DLX treatment $(6.22 \pm 1.92$ at baseline and $2.78 \pm 2.44$ at 3 months). The mean NRS numbness score was $6.34 \pm 2.01$ at baseline and $4.13 \pm 2.443$ months after DLX initiation in all patients with numbness $(\mathrm{n}=23)$. The NRS numbness scores decreased from $6.33 \pm 1.68$ to $3.56 \pm 2.01$ in the E group and from $6.40 \pm 3.21$ to $6.20 \pm 2.95$ in the $\mathrm{N}$ group (Fig. 1A, B). The mean NRS pain and numbness scores significantly decreased in the E group $(p<0.01)$ (Fig. 1A). The intensity of symptoms at baseline and 3 months following DLX initiation is presented in Fig. 2. DLX reduced the intensity of postsurgical chronic pain. At baseline, two patients had severe postsurgical chronic pain, and seven had moderate postsurgical chronic pain. Three months after DLX initiation, three had moderate postsurgical chronic pain, four had mild postsurgical chronic pain, and two had no postsurgical chronic pain (Fig. 2A). In addition, DLX relatively mitigated the intensity of postsurgical numbness. At baseline, 10 patients had severe postsurgical chronic numbness, and 13 had moderate postsurgical chronic numbness. Three months after DLX initiation, three had severe postsurgical chronic numbness, eight had moderate postsurgical chronic numbness, and 12 had
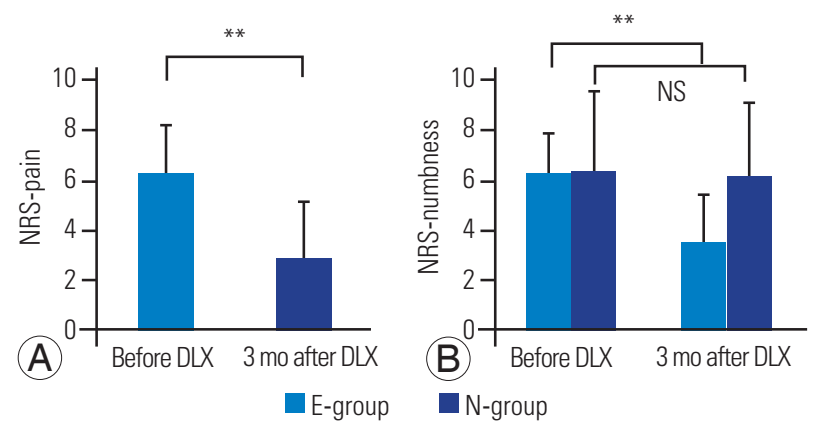

Fig. 1. Post-surgical chronic pain and numbness assessed by the Numeric Rating Scale (NRS). (A) The mean NRS pain score $(n=9)$. Duloxetine (DLX) reduced post-surgical chronic pain by more than 2 points on the NRS. All nine patients with post-surgical chronic pain were in the E group. There was a significant decrease in the NRS pain scores after 3 months of DLX treatment compared with baseline (before DLX administration). (B) The mean NRS post-surgical chronic numbness scores (total $n=23$ ). The E group patients with numbness $(n=18)$ exhibited a significant decrease in symptoms 3 months after DLX treatment initiation, while the $N$ group patients with numbness $(n=5)$ showed no symptom relief. NS, no significant difference. ${ }^{* *} p<0.01$. 
mild postsurgical chronic numbness (Fig. 2B). There were no patients with complete relief of numbness after DLX treatment.

The proportion of patients with a marked pain response to DLX (i.e., very much improved) was $66.7 \%$ (six of nine patients with postsurgical chronic pain), and the proportion of those with much improved pain was 33.3\% (three out of nine patients). With regard to the reduction
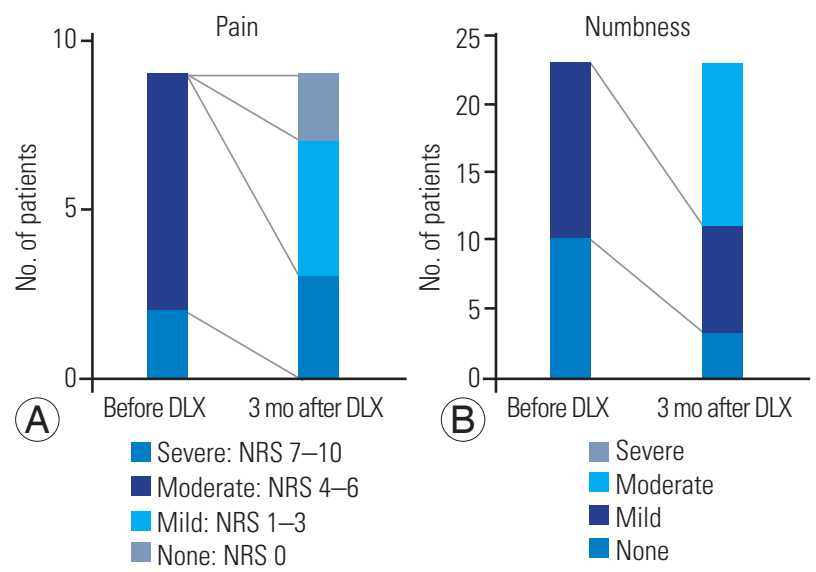

Fig. 2. The intensity of each post-surgical chronic symptom. Pain and numbness were evaluated with the Numeric Rating Scale (NRS) as follows: NRS score $\geq 7$ as severe, NRS score of 4-6 as moderate, NRS score of $1-3$ as mild, and NRS score of 0 as none. (A) The change in pain intensity before and 3 months after duloxetine (DLX) treatment. Two patients had complete pain relief (NRS=0) after 3 months of DLX treatment. (B) The change in the intensity of numbness before and 3 months after DLX treatment.
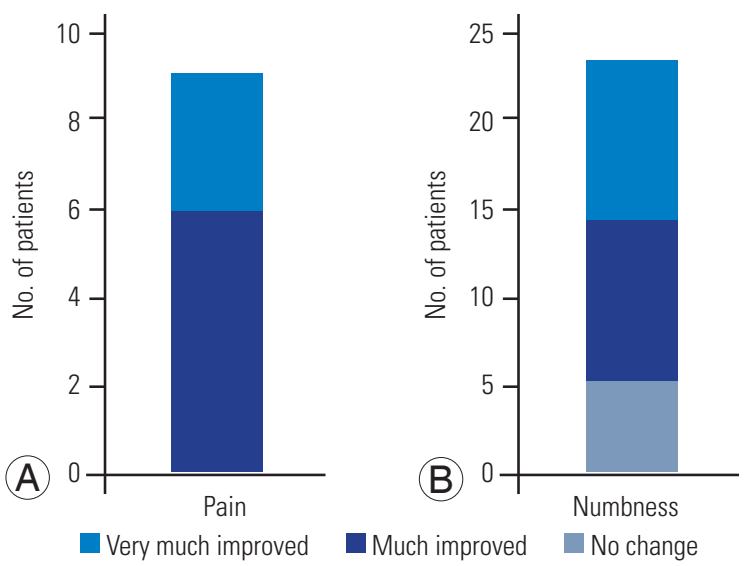

Fig. 3. (A, B) Assessment of improvement in each symptom. The degree of symptom relief was defined as follows: symptoms with a decrease in the Numeric Rating Scale (NRS) score $\geq 2$ as much improved, a decrease in the NRS score more than $50 \%$ compared with baseline or NRS $\leq 2$ after 3 months of treatment as very much improved, and a decrease in the NRS score $\leq 1$ as no change. Among the patients with post-surgical chronic pain, 33\% showed a very much improved change, and $66 \%$ showed a much improved change. For post-surgical chronic numbness, $39.1 \%$ patients each showed very much improved and much improved changes (nine patients each), while $21.7 \%$ exhibited no change in numbness. in postsurgical chronic numbness, the proportions of patients with very much improved and much improved numbness were $39.1 \%$ and $39.1 \%$, respectively (nine of 23 cases each), whereas five patients (21.7\%) exhibited no change after 3 months of DLX treatment (Fig. 3).

The mean postoperative duration before DLX administration in each group was $50.5 \pm 60.8$ months (range, 3-180 months) in the E group and $45.8 \pm 44.6$ months (range, $10-120$ months) in the $\mathrm{N}$ group. No statistically significant difference was observed in this duration. There were three patients with a postoperative duration of 15 years, and DLX was effective in all of them. With regard to the maintenance dose of DLX per day, $47 \%$ of patients in the E group $(\mathrm{n}=9)$ received $40 \mathrm{mg} /$ day, $32 \%(\mathrm{n}=6)$ received $60 \mathrm{mg} /$ day, and $21 \%(\mathrm{n}=4)$ received $20 \mathrm{mg} /$ day, whereas all patients in the $\mathrm{N}$ group $(\mathrm{n}=5)$ received $60 \mathrm{mg} /$ day. In terms of ongoing concomitant prescriptions with DLX in this study, 14 patients received pregabalin, and five patients received tramadol. No significant difference was observed in the distribution of these patients between the $\mathrm{E}$ and $\mathrm{N}$ groups (Table 2). Among all participants, four had a history of diabetes, all of whom were in the E group $(p<0.05)$ (Table 2).

Although DLX reduced postsurgical pain and numbness in 19 patients (79.2\%) in this analysis, DLX exhibited no efficacy in postsurgical numbness in five of 24 patients. The details of these five patients, who were all in the $\mathrm{N}$ group, are presented in Table 3. Among them, two who underwent multiple back operations (i.e., several lumbar decompressions and/or fusion surgeries) had tandem spinal stenosis at another spinal site, and one had carpal tunnel syndrome after cervical laminoplasty (Table 3).

\section{Discussion}

Acute postoperative pain is caused by several mechanisms, such as nociceptive, neuropathic, and inflammatory mechanisms [18]. Hence, spinal surgeries are rated among the top six procedures that elicit intense postsurgical pain for the initial few days [19], and adequate and energetic postsurgical pain management is mandatory to prevent the development of postsurgical chronic pain and peripheral/central sensitization. However, most patients who underwent spinal surgery already had preexisting pain which, along with preoperative long-term analgesic use, might alter their pain perception, thereby complicating their pain management. Pregabalin, which is a struc- 


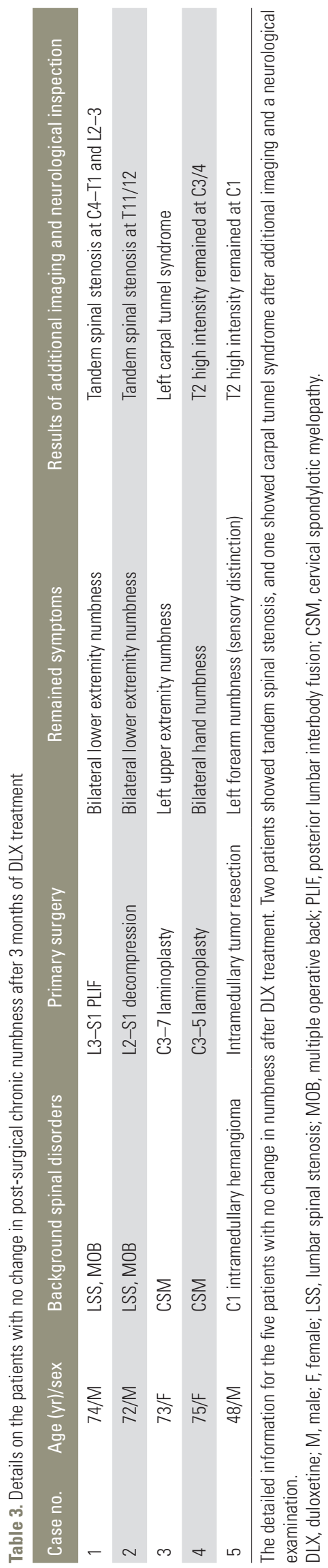

tural analog of $\gamma$-aminobutyric acid and binds to the $\alpha 2 \delta$ 1 and $\alpha 2 \delta-2$ auxiliary subunits of the presynaptic calcium channels, is widely prescribed to achieve adequate pain relief following elective spinal surgery. Several previous studies have reported the effectiveness of perioperative pregabalin administration in reducing postoperative pain $[20,21]$. Urban et al. [22] reported that pregabalin administration did not reduce perioperative opioid consumption and did not improve acute postoperative pain management. Although pregabalin had some positive effects on postoperative residual symptoms, we frequently encountered patients in the clinic with refractory postsurgical chronic numbness with pregabalin prescriptions. Therefore, a further therapeutic modality to effectively treat postsurgical chronic numbness is desired. In this study, we retrospectively evaluated the effect of DLX on postsurgical chronic residual pain and numbness following spinal surgery and found positive effects. DLX is an SNRI agent assumed to augment descending pain inhibitory pathway by inhibiting the reuptake of noradrenalin and serotonin into synaptosomes in the spinal cord. Several analgesic mechanisms of DLX have been proposed through animal neuropathic pain models: DLX could (1) increase spinal noradrenalin and serotonin levels, (2) activate spinal 5-HT2A or a 2-noradrenergic receptors, and (3) inhibit neuronal $\mathrm{Na}^{+}$currents in the spinal cord [23].

In terms of the efficacy of DLX for spinal surgeryrelated symptoms, although several reports indicated the potential of DLX to effectively treat postoperative symptoms as a perioperative intervention, thus far, there are no reports of its positive effect on postsurgical chronic neuropathic disorders following spinal surgery. There are several reports of randomized, double-blind, controlled studies advocating the positive effects of perioperative DLX prescriptions for postsurgical analgesia. Hyer et al. [24] elucidated that combination therapy with an opioid and DLX administered 2 weeks before spine surgery could improve postoperative pain, assist with maintaining function, and reduce the affect intensity compared with the opioid and placebo group. Bedin et al. [10] elucidated the adjunct effect of DLX for postoperative analgesia and the positive function of reducing opioid consumption following elective spine surgery. Attia and Mansour [12] reported significant pain relief after lumbar laminectomy with combined DLX and etoricoxib treatment. In addition to these positive effects of DLX combined with other analgesics as perioperative interventions, in a randomized, 
double-blind, placebo-controlled phase 3 trial, Konno et al. [7] recently reported that DLX monotherapy could improve pain severity in Japanese patients with chronic low back pain. The current study, albeit a retrospective analysis, was the first study to indicate the effectiveness of DLX for both postsurgical chronic myelopathic disorders of pain and numbness in the chronic stage following surgery (mean duration after surgery, 35.5 \pm 57.0 months). The results of this study suggest the effectiveness of DLX on postsurgical chronic neuropathic pain. DLX has been reported to be effective for diabetic neuropathy and chemotherapy-induced neuropathic pain in cancer-bearing patients; however, there is no report of its effectiveness on postsurgical chronic neuropathic pain following spinal surgery. Although there is no report of the reinforcement of temporal summation in patients with prolonged pain after spinal surgery, it has been reported that there are some populations of low back pain patients who were strongly involved with central sensitization [25]. In a previous research on diabetic neuropathy, DLX was proven to be more effective in cases with increased temporal summation and decreased conditioned pain modulation, improving temporal summation and conditioned pain modulation [26]. In an animal experimental study, gabapentin administration onto rats with L5 nerve root ligation model ceased to be effective from 4 weeks after ligation [27]. Moreover, pregabalin could not reactivate the endogenous analgesia systems under the capsaicininduced nociceptive stimuli at 6 weeks after L5 nerve ligation, whereas SNRIs, such as amitriptyline or DLX, could reactivate them [28]. Although it was actually unknown whether patients enrolled in this study had exhibited central sensitization, the mechanism of DLX effectiveness on postsurgical chronic neuropathic disorders might be based on the improvement of intrinsic analgesia systems in patients following spinal surgery in whom the descending pain inhibitory system was attenuated.

Although DLX seemed to have some effects on chronic neuropathic disorders after spine and spinal cord surgery, there were indeed several cases with persistent postsurgical chronic numbness for which no intervention was effective. In this study, five patients with postoperative chronic numbness exhibited no symptom relief after DLX initiation. Minute neurological examinations revealed that two patients had tandem spinal stenosis, and one had entrapment peripheral neuropathy. The morbidity rate of tandem spinal stenosis among patients with spinal ste- nosis was approximately $60 \%$, which is often overlooked [29]. Double crush syndrome, which was first described in 1973, has been frequently reported to have a higher prevalence of cervical radiculopathy, especially in patients with carpal tunnel syndrome, compared with the general population [30]. When postsurgical chronic numbness is experienced after spine and spinal cord surgery, spine surgeons should pay attention to the possibility of these concomitant neurological disorders.

This study has some limitations. First, the retrospective design of this study unavoidably lowered the evidence level. Second, the sample size was quite small, and there was no control group. Finally, the population enrolled in this study was heterogeneous in terms of both underlying spinal disorders and concomitant drug use. However, our strength was that this is the first study to analyze the effectiveness of DLX in postsurgical chronic pain and numbness. Future prospective studies with larger samples and a randomized, controlled setting should be considered to evaluate the effectiveness of DLX in postsurgical chronic neuropathic disorders.

\section{Conclusions}

This study indicated that DLX reduced chronic symptoms of postsurgical chronic neuropathic disorders (i.e., pain and numbness). Although postsurgical numbness was often intractable, strong neurological examinations should be reconsidered to increase awareness of the signs of occult neurological disorders. DLX reduced postsurgical chronic pain (efficacy rate $=100 \%$ ) and numbness $(78.3 \%)$ in certain patients. Further investigations are required to determine the optimal use of DLX.

\section{Conflict of Interest}

No potential conflict of interest relevant to this article was reported.

\section{Author Contributions}

OT designed the study and wrote the initial draft of the manuscript. MM, MN, and KW was responsible for designing the study protocol and recruiting participants. SK played an important role in interpreting the result and contributed to the preparation of the manuscript. SN, SS, EO, NF, and MY contributed to analysis and interpreta- 
tion of data and assisted in the preparation of the manuscript. KW was responsible for all working related to this submission as corresponding author. Also, all authors approved the final version manuscript and agreed to be accountable for all aspects of the work.

\section{References}

1. Cho JH, Lee JH, Song KS, Hong JY. Neuropathic pain after spinal surgery. Asian Spine J 2017;11:642-52.

2. Haanpaa M, Attal N, Backonja M, et al. NeuPSIG guidelines on neuropathic pain assessment. Pain 2011;152:14-27.

3. Khosravi MB, Azemati S, Sahmeddini MA. Gabapentin versus naproxen in the management of failed back surgery syndrome; a randomized controlled trial. Acta Anaesthesiol Belg 2014;65:31-7.

4. Baber Z, Erdek MA. Failed back surgery syndrome: current perspectives. J Pain Res 2016;9:979-87.

5. Wernicke JF, Pritchett YL, D'Souza DN, et al. A randomized controlled trial of duloxetine in diabetic peripheral neuropathic pain. Neurology 2006;67:141120.

6. Arnold LM, Rosen A, Pritchett YL, et al. A randomized, double-blind, placebo-controlled trial of duloxetine in the treatment of women with fibromyalgia with or without major depressive disorder. Pain 2005;119:5-15.

7. Konno S, Oda N, Ochiai T, Alev L. Randomized, double-blind, placebo-controlled phase III trial of duloxetine monotherapy in Japanese patients with chronic low back pain. Spine (Phila Pa 1976) 2016;41:1709-17.

8. Enomoto H, Fujikoshi S, Tsuji T, Sasaki N, Tokuoka H, Uchio Y. Efficacy of duloxetine by prior NSAID use in the treatment of chronic osteoarthritis knee pain: a post hoc subgroup analysis of a randomized, placebo-controlled, phase 3 study in Japan. J Orthop Sci 2018;23:1019-26.

9. Ho KY, Tay W, Yeo MC, et al. Duloxetine reduces morphine requirements after knee replacement surgery. Br J Anaesth 2010;105:371-6.

10. Bedin A, Caldart Bedin RA, Vieira JE, Ashmawi HA. Duloxetine as an analgesic reduces opioid consumption after spine surgery: a randomized, double-blind, controlled study. Clin J Pain 2017;33:865-9.

11. Prabhala T, Sabourin S, DiMarzio M, Gillogly M,
Prusik J, Pilitsis JG. Duloxetine improves spinal cord stimulation outcomes for chronic pain. Neuromodulation 2019;22:215-8.

12. Attia JZ, Mansour HS. Perioperative Duloxetine and Etoricoxibto improve postoperative pain after lumbar laminectomy: a randomized, double-blind, controlled study. BMC Anesthesiol 2017;17:162.

13. Treede RD, Jensen TS, Campbell JN, et al. Neuropathic pain: redefinition and a grading system for clinical and research purposes. Neurology 2008;70:1630-5.

14. Finnerup NB, Haroutounian S, Kamerman P, et al. Neuropathic pain: an updated grading system for research and clinical practice. Pain 2016;157:1599-606.

15. Farrar JT, Young JP Jr, LaMoreaux L, Werth JL, Poole RM. Clinical importance of changes in chronic pain intensity measured on an 11-point numerical pain rating scale. Pain 2001;94:149-58.

16. Serlin RC, Mendoza TR, Nakamura Y, Edwards KR, Cleeland CS. When is cancer pain mild, moderate or severe?: grading pain severity by its interference with function. Pain 1995;61:277-84.

17. Dworkin RH, Turk DC, Wyrwich KW, et al. Interpreting the clinical importance of treatment outcomes in chronic pain clinical trials: IMMPACT recommendations. J Pain 2008;9:105-21.

18. Mathiesen O, Dahl B, Thomsen BA, et al. A comprehensive multimodal pain treatment reduces opioid consumption after multilevel spine surgery. Eur Spine J 2013;22:2089-96.

19. Gerbershagen HJ, Aduckathil S, van Wijck AJ, Peelen LM, Kalkman CJ, Meissner W. Pain intensity on the first day after surgery: a prospective cohort study comparing 179 surgical procedures. Anesthesiology 2013;118:934-44.

20. Yu L, Ran B, Li M, Shi Z. Gabapentin and pregabalin in the management of postoperative pain after lumbar spinal surgery: a systematic review and metaanalysis. Spine (Phila Pa 1976) 2013;38:1947-52.

21. Khurana G, Jindal P, Sharma JP, Bansal KK. Postoperative pain and long-term functional outcome after administration of gabapentin and pregabalin in patients undergoing spinal surgery. Spine (Phila $\mathrm{Pa}$ 1976) 2014;39:E363-8.

22. Urban MK, Labib KM, Reid SC, et al. Pregabalin did not improve pain management after spinal fusions. HSS J 2018;14:41-6.

23. De Oliveira Filho GR, Kammer RS, Dos Santos HC. 
Duloxetine for the treatment acute postoperative pain in adult patients: a systematic review with metaanalysis. J Clin Anesth 2020;63:109785.

24. Hyer L, Scott C, Mullen CM, McKenzie LC, Robinson JS. Randomized double-blind placebo trial of duloxetine in perioperative spine patients. J Opioid Manag 2015;11:147-55.

25. Correa JB, Costa LO, de Oliveira NT, Sluka KA, Liebano RE. Central sensitization and changes in conditioned pain modulation in people with chronic nonspecific low back pain: a case-control study. Exp Brain Res 2015;233:2391-9.

26. Yarnitsky D, Granot M, Nahman-Averbuch H, Khamaisi M, Granovsky Y. Conditioned pain modulation predicts duloxetine efficacy in painful diabetic neuropathy. Pain 2012;153:1193-8.
27. Kimura M, Eisenach JC, Hayashida K. Gabapentin loses efficacy over time after nerve injury in rats: role of glutamate transporter-1 in the locus coeruleus. Pain 2016;157:2024-32.

28. Ito $S$, Suto T, Saito $S$, Obata H. Repeated administration of duloxetine suppresses neuropathic pain by accumulating effects of noradrenaline in the spinal cord. Anesth Analg 2018;126:298-307.

29. Overley SC, Kim JS, Gogel BA, Merrill RK, Hecht AC. Tandem spinal stenosis: a systematic review. JBJS Rev 2017;5:e2.

30. Schmid AB, Coppieters MW. The double crush syndrome revisited: a Delphi study to reveal current expert views on mechanisms underlying dual nerve disorders. Man Ther 2011;16:557-62. 